\title{
Knee Stability following Posterior-Stabilized Total Knee Arthroplasty: Comparison of Medial Preserving Gap Technique and Measured Resection Technique
}

\author{
Masanori Tsubosaka, MD ${ }^{1}$ Hirotsugu Muratsu, MD² Naoki Nakano, MD ${ }^{1}$ Tomoyuki Kamenaga, MD ${ }^{1}$ \\ Yuichi Kuroda, MD ${ }^{1}$ Takao Inokuchi, MD² Hidetoshi Miya, MD² Ryosuke Kuroda, MD \\ Tomoyuki Matsumoto, MD ${ }^{1}$
}

${ }^{1}$ Department of Orthopedic Surgery, Kobe University Graduate School of Medicine, Kobe, Japan

2 Department of Orthopedic Surgery, Steel Memorial Hirohata Hospital, Himeji, Japan

Address for correspondence Hirotsugu Muratsu, MD, Department of Orthopedic Surgery, Steel Memorial Hirohata Hospital, 3-1, Yumesakicho, Hirohata-ku, Himeji, Hyogo, 671-1122, Japan (e-mail: hiromuratsu@gmail.com).

J Knee Surg 2023;36:95-104.

\begin{abstract}
Keywords

- total knee arthroplasty

- postoperative knee stability

- posterior-stabilized

- medial preserving gap technique

- measured resection technique

- soft tissue balance

Novel medial preserving gap technique (MPGT) focuses on medial compartment stability and allows lateral physiological laxity. This study aimed to compare the MPGT with the measured resection technique (MRT) to determine which provides better postoperative knee stability after posterior-stabilized total knee arthroplasty (PS-TKA). Primary PS-TKA, using either MPGT $(n=65)$ or MRT $(n=65)$, was performed in 130 patients with varus knee osteoarthritis. Postoperative knee stabilities at extension and flexion were assessed using varus-valgus stress radiographs and stress epicondylar view, respectively (at 1 month, 6 months, 1 year, and 3 years postoperatively). The distance between the femoral prosthesis and polyethylene insert was measured on each medial and lateral side, defined as the medial joint opening (MJO) and lateral joint opening (LJO), respectively. Decreasing MJO or LJO translated to increasing postoperative stability. The femoral external rotation angle was compared between the two surgical techniques; postoperative knee stability was also compared between the medial and lateral compartments, as well as the surgical techniques. A significant difference was found in the femoral external rotation angle between MPGT $(4.2 \pm 0.2$ degrees $)$ and MRT $(3.6 \pm 0.1$ degrees, $p<0.01)$. Postoperative MJOs both at extension and flexion were significantly smaller than LJOs using MPGT and MRT at all time points $(p<0.05)$. MJOs and LJOs at extension using MPGT were significantly smaller than those when using MRT, at 1 and 3 years postoperatively $(p<0.05)$. Furthermore, MJOs at flexion using MPGT were significantly smaller than those when using MRT at 6 months, 1 year, and 3 years postoperatively $(p<0.05)$. MPGT provided higher postoperative medial knee stability than MRT both at extension and flexion, even at 3 years after PS-TKA. This suggests that this newly developed surgical technique is a more feasible option than MRT for the preservation of postoperative medial knee stability.
\end{abstract}

received

January 21, 2021

accepted after revision

April 1, 2021

article published online

May 15, 2021 (c) 2021. Thieme. All rights reserved.

Thieme Medical Publishers, Inc., 333 Seventh Avenue, 18th Floor, New York, NY 10001, USA
DOI https://doi.org/ 10.1055/s-0041-1729968. ISSN 1538-8506. 
Measured resection technique (MRT) and gap balancing technique are two common surgical methods for total knee arthroplasty (TKA). ${ }^{1,2}$ The gap balancing technique is more effective for achieving an equalized rectangular gap, which is an equal soft tissue balance, at extension and flexion than MRT. ${ }^{3}$ However, the risk of medial instability arises in many cases when aiming for the perfect ligament balance at extension because of excessive medial release. The medial instability after TKA causes postoperative knee pain ${ }^{4}$ and nonphysiologic movement of the knee. ${ }^{5}$

To overcome the concerns of the gap balancing technique, a novel medial preserving gap technique (MPGT) has been developed and recently performed. MPGT focuses on medial knee stability, essentially making not equal rectangular gaps but equal trapezoidal gaps in extension and flexion. ${ }^{6}$ It also provides quantitative gap balancing technique using tensor measurements independent of surgeons' feels and experiences. Posterior-stabilized (PS) TKA with MPGT provides more consistent intraoperative soft tissue balance during knee flexion than MRT. ${ }^{7}$ However, postoperative knee stability especially in medial compartment, which was reported as an important factor associated with postoperative ambulation function and patient satisfaction, ${ }^{8}$ has not been investigated in the knees after PS-TKA with MPGT.

The present study aimed to determine whether MPGT could provide the higher postoperative medial knee stability, which was essential for clinical outcome, compared with MRT as long as 3 years after PS-TKA. It was hypothesized that postoperative medial knee stability both at extension and flexion with MPGT was better than that with MRT after PSTKA.

\section{Materials and Methods}

\section{Patients}

This retrospective study included 130 patients with varus knee osteoarthritis (OA) who met the criteria and underwent primary PS-TKA (NexGen LPS Flex; Zimmer, Warsaw, IN) between 2008 and 2012. Varus knee OA was defined as hipknee-ankle (HKA) $\geq 2$ degrees in varus from full-limb radiograph. ${ }^{9}$ Inclusion criteria were substantial pain, loss of function due to knee $\mathrm{OA}$, and ineffectiveness of conservative treatment including rehabilitation, medication, and intra-articular injection of hyaluronic acid or steroids. Exclusion criteria were valgus knee deformity, severe bony defects a requiring bone graft or augmentation, previous knee surgery, revision TKA, and active knee joint infection. Patients undergoing cruciate-retaining TKA were also excluded; thus, 135 patients were excluded. PS-TKA using MPGT was performed in 65 patients (MPGT group) between 2010 and 2012, whereas the control group comprised 65 patients who underwent PS-TKA using MRT (MRT group) between 2008 and 2010. The mean HKAs of the MPGT and MRT groups were 13.1 degrees in varus (range: 2.424.5 degrees) and 11.4 degrees in varus (range: 4.225.6 degrees), respectively, and there was no significant difference between the two groups. Other patient demographic data are shown in - Table 1. Each surgery was performed by the same surgeon, the senior author (H.M.). The hospital ethics committee approved the study protocol, and the patients provided written informed consent for their participation.

\section{Offset Repo-Tensor}

The Offset Repo-Tensor (OFR tensor) (Zimmer, Warsaw, IN) consists of three parts: an upper seesaw plate, a lower platform plate with a spike, and an extraarticular main body, as previously described. ${ }^{10-13}$ This device facilitates the measurement of joint ligament balance and joint center gap, both before and after femoral trial component placement, while applying a constant joint distraction force. The PS type seesaw plate has a post at the proximal center to fit the intercondylar space and the cam of the femoral trial component in PS-TKA. This post-cam mechanism controls the tibiofemoral position in both the coronal and sagittal planes, reproducing the joint constraint and alignment after the components are implanted. Joint distraction forces ranging from $20 \mathrm{lbs}(9.1 \mathrm{~kg})$ to $60 \mathrm{lbs}(27.2 \mathrm{~kg})$ can be exerted between the seesaw and platform plates using a specially made torque driver that can change the maximum torque value within an error for joint distraction within $\pm 3 \%$. This torque driver is placed on a rack that contains a rack-andpinion mechanism along the extraarticular main body. The appropriate torque is applied to generate the required distraction force, and two scales are evaluated according to the tensor measurement: the angle (degree, positive value in

Table 1 Patient demographic data

\begin{tabular}{|c|c|c|c|}
\hline & Medial preserving gap technique & Measured resection technique & p-Value \\
\hline Cases & 65 & 65 & \\
\hline Age at surgery $(\mathrm{y})$ & $74.3 \pm 5.7$ & $73.4 \pm 6.4$ & n.s. \\
\hline Sex (male/female) & $9 / 56$ & $7 / 58$ & n.s. \\
\hline Weight $(\mathrm{kg})$ & $58.5 \pm 10.8$ & $57.8 \pm 8.7$ & n.s. \\
\hline BMI $\left(\mathrm{kg} / \mathrm{m}^{2}\right)$ & $25.6 \pm 3.9$ & $25.4 \pm 3.6$ & n.s. \\
\hline $\begin{array}{l}\text { Active flexion angle (degree) } \\
\text { Active extension angle (degree) }\end{array}$ & $\begin{array}{l}107.4 \pm 17.2 \\
-7.8 \pm 9.0\end{array}$ & $\begin{array}{l}107.5 \pm 20.3 \\
-5.7 \pm 10.9\end{array}$ & $\begin{array}{l}\text { n.s. } \\
\text { n.s. }\end{array}$ \\
\hline
\end{tabular}

Abbreviations: BMI, body mass index; n.s., not significant.

Data are shown as mean \pm standard deviation. 
varus ligament balance) between the seesaw and the platform plates, and the distance ( $\mathrm{mm}$, joint center gap) between the center midpoints of the upper seesaw plate and the proximal tibial cut. By measuring these angular deviations and distances under a constant joint distraction force, both the angle and joint center gap could be measured. The smallest scales of these measurements are 1 degree and $1 \mathrm{~mm}$, respectively.

\section{Surgical Procedures}

\section{MPGT}

After inflating the tourniquet, the surgeries were performed with a medial parapatellar approach. First, both the anterior cruciate ligament and posterior cruciate ligament were resected. Then, a distal femoral osteotomy was performed perpendicular to the mechanical axis of the femur in the coronal plane using an intramedullary resection guide, according to preoperative long-leg radiographs. A proximal tibial osteotomy was then performed with a $10-\mathrm{mm}$ bone resection from lateral tibial plateau and perpendicular to the mechanical axis in the coronal plane and with 7 degrees of posterior inclination according to the manufacture's instruction along the sagittal plane using an extramedullary guide. No bony defects were observed along the eroded medial tibial plateau. The medial soft tissue release was limited by femoral and tibial osteophyte removal and deep medial collateral ligament (MCL) release within $1 \mathrm{~cm}$ from medial tibial plateau until a $10-\mathrm{mm}$ spacer block could be inserted. Lateral physiological laxity was allowed to avoid excessive medial releases. Neutral alignment was confirmed with each cut of the distal femur and proximal tibia by ensuring that the alignment rod was connected to a spacer block located at the center of the femoral head and ankle joint.

The OFR tensor was placed with the lower platform plate located at the center of the proximal tibia. Thereafter, knee gap was measured between the osteotomized femoral and tibial surfaces at extension, and between posterior femoral condyles and the osteotomized tibial surface at flexion. A distraction force of $40 \mathrm{lbs}$ was loaded, and the varus ligament balance and joint center gap were measured both at extension and flexion. The joint distraction force was loaded several times until the joint center gap remained constant to reduce the error that could result from creep elongation of the surrounding soft tissue. We confirmed that the bone was not compressed or deformed during distraction force application. During each measurement, the thigh was held and the knee was aligned along the sagittal plane to eliminate the external load on the knee both at extension and flexion.

The main feature of MPGT consists of planning the posterior femoral condylar osteotomy. According to a previous report, the differences in varus ligament balance between extension and flexion were consistent across different joint distraction forces. ${ }^{13}$ These findings suggested that the differences in varus ligament valance between extension and flexion could be an index for the external rotation angle of the posterior femoral condyle osteotomy. Thus, the femoral external rotation angle was determined based on the differ- ences in varus ligament balance between extension and flexion (-Fig. 1A). The difference in the joint center gap between extension and flexion was used to determine both the femoral component size and the corresponding amount of posterior femoral condyle resection. The original femoral anteroposterior size was measured using the anterior reference technique beforehand, while the gap difference between extension and flexion was compared with the distal thickness of the femoral component; thereafter, a femoral component of appropriate size was selected to reduce the gap difference between extension and flexion (-Fig. 1B). After each bony resection, the tensor and femoral trial components were fixed and the patellofemoral joint was reduced by temporarily suturing the medial parapatellar arthrotomy. The final soft tissue balance, including the joint center gap and varus ligament balance, was then measured with the knee from extension to flexion. The thickness of the polyethylene insert was determined based on the joint center gap with a femoral trial component in place at extension, with $40 \mathrm{lbs}$ of joint distraction forces. ${ }^{6}$ Finally, a polyethylene insert was implanted, as well as a NexGen component with cement.

\section{MRT}

Distal femoral and proximal tibial osteotomies were performed in the same manner as previously mentioned in MPGT. No bony defects were noted along the eroded medial tibial plateau in any of the cases. At this point, the osteophytes were removed. Following confirmation of neutral alignment of the knee with the spacer block and alignment rod, a posterior femoral cut was made using the anterior referencing technique. Femoral external rotation was usually set at 3 or 5 degrees relative to the posterior condylar axis, ${ }^{14}$ with reference to the Whiteside's line $e^{15,16}$ and the epicondylar axis, ${ }^{17}$ as well as the condylar twist angle, measured using the preoperative computed tomography images. ${ }^{18}$ Thereafter, we appropriately released the soft tissues (deep layer MCL, medial, and posteromedial capsule) along the medial knee structures. The soft tissue balance was finally adjusted in coronal plane by additional soft tissue release (e.g., superficial layer of MCL) to obtain equal extension flexion gap. The thickness of the polyethylene insert was determined based on the joint center gap with a femoral trial component in place at extension, with 40 lbs of joint distraction forces. ${ }^{6}$ Subsequently, a NexGen prosthesis was implanted using cement, as well as a polyethylene insert.

\section{Postoperative Radiographic Assessment of Components}

Postoperative anteroposterior radiographs of the lower limb were obtained for evaluation of the coronal femoral component angle (FCA) and tibial component angle (TCA). Lateral radiographs of the lower limb were obtained for the evaluation of the sagittal TCA. FCA was defined as the angle between the femoral mechanical axis and the joint surface line of the femoral implant. TCA was defined as the angle between the tibial mechanical axis and the base plate of the tibial implant. 

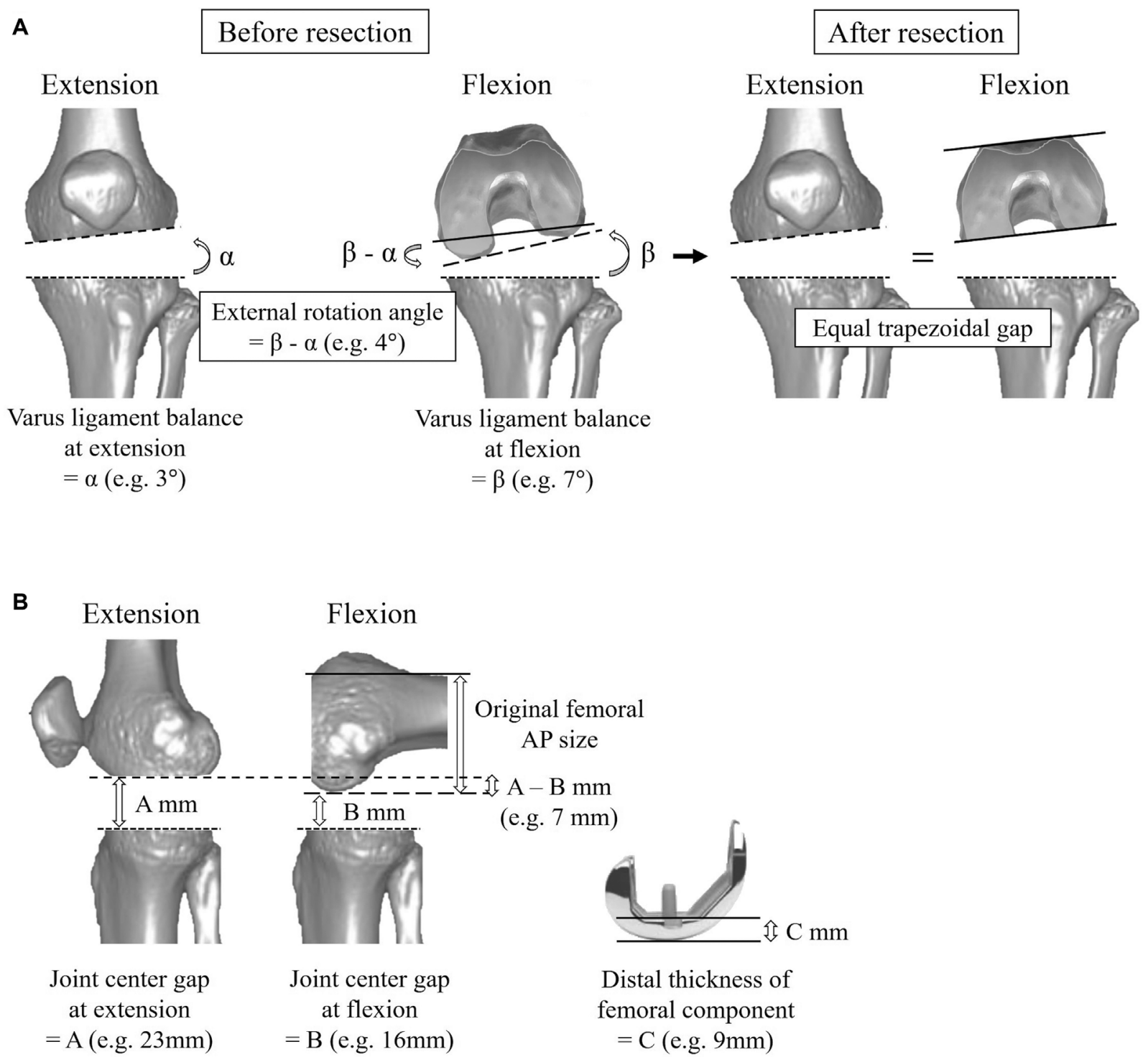

Distal thickness of femoral component $=\mathrm{C}(\mathrm{e} . \mathrm{g} .9 \mathrm{~mm})$

Fig. 1 Determination method of femoral external rotation angle and thickness of posterior femoral condylar resection. (a) The external rotation angle of the posterior femoral condyle is determined within the range of 0 to 7 degrees with reference to the difference in varus ligament balance between extension and flexion. This angle is independent from joint distraction forces. (e.g., varus ligament balances at extension and flexion were 3 degrees $(=\alpha)$ and 7 degrees $(=\beta)$, respectively. External rotation angle $=7-3$ degrees $=4$ degrees). (b) The thickness of posterior femoral condylar resection is defined as the difference in joint center gap between extension and flexion. The original femoral anteroposterior (AP) size is measured using the anterior reference technique beforehand, while the gap difference between extension and flexion (A-B) is compared with the distal thickness of the femoral component (C); thereafter, a femoral component of appropriate size is selected. Index for determination of femoral component size was described as follows; A-B $<$ C: Femoral component with larger AP size should be selected compared with original femoral AP size. (e.g., 23-16mm <9mm: Femoral component with larger AP size than original femoral AP size by $2 \mathrm{~mm}$ should be selected.) A$\mathrm{B}=\mathrm{C}$ : Femoral component with the original femoral AP size should be selected. A-B $>C$ : Femoral component with smaller AP size should be selected.

\section{Postoperative Knee Stability Measurements}

Postoperative knee stabilities at extension and flexion were assessed using stress radiographies at 1 month, 6 months, 1 year, and 3 years after TKA. Postoperative knee stability at extension was assessed using varus-valgus stress X-ray with a Telos SE arthrometer (FaTelos; Medizinisch-Technische, Griesheim, Germany) following a previously reported method. ${ }^{19}$ Valgus or varus force of $10 \mathrm{~kg}$ was applied immediately above the joint on the lateral or medial femoral condyle, whereas the proximal thigh and middle leg were held by the counter support at 10 degrees of flexion. Joint separation distance $(\mathrm{mm})$ between the lowest point of femoral prosthesis and the line in contact with the lower surface of tibial prosthesis at medial and lateral compartments was measured. The distance was adjusted using magnification based on the keel width of the tibial prosthesis. The distance between the lowest point of femoral prosthesis and the upper surface of the polyethylene insert was calculated and defined as medial joint opening (MJO) and lateral joint opening (LJO). The joint opening $(\mathrm{mm})$ was calculated as follows: joint opening $=$ joint separation distance - insert thickness. On varus-valgus stress radiographies, the angles between the 

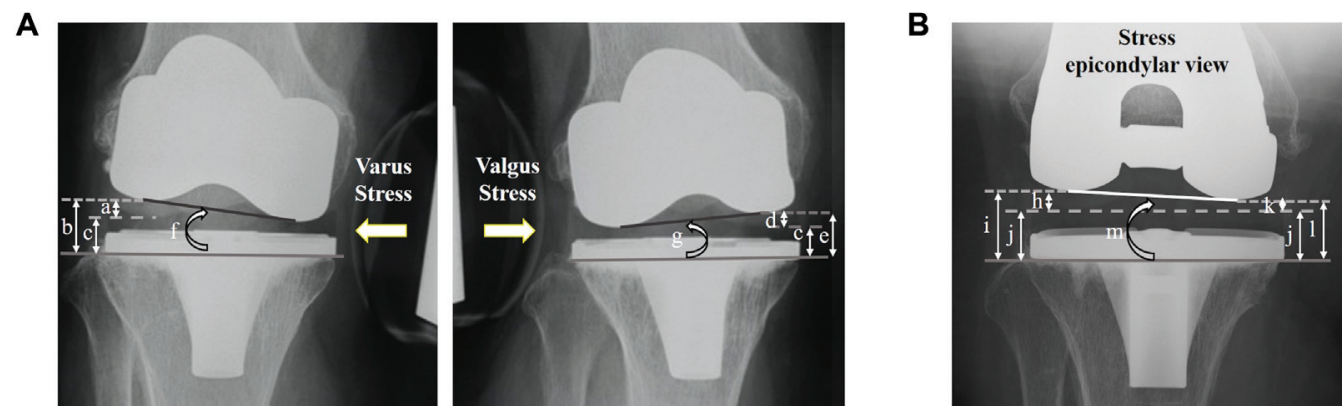

Fig. 2 Postoperative knee stability measurements using stress radiographies. (A) Extension knee stability at lateral and medial sides was assessed using varus-valgus stress radiographies. a, lateral joint opening; b, lateral joint separation distance; c, insert thickness; $d$, medial joint opening; e, medial joint separation distance; $f$, varus angle at extension; $g$, valgus angle at extension; varus ligament balance at extension = ( $f$ ) (g). (B) Flexion knee stability was assessed using stress epicondylar view radiograph. $\mathrm{H}$, lateral joint opening; i, lateral joint separation distance; j, insert thickness; k, medial joint opening; I, medial joint separation distance; m, varus angle at flexion =varus ligament balance at flexion.

line in contact with the medial and lateral lowest points of femoral prosthesis and the line in contact with the lower surface of the tibial prosthesis were measured. These varus and valgus angles indicated the degree of lateral and medial laxities, respectively. "Varus ligament balance at extension" was defined as "varus angle at extension - valgus angle at extension," which means lateral laxity (positive value in varus), following a previously reported $\operatorname{method}^{20}$ (- Fig. 2A).

Postoperative knee stability at flexion was assessed using the stress epicondylar view with $1.5 \mathrm{~kg}$ weight at the ankle, ${ }^{21,22}$ which enabled to visualize the posterior condylar axis and the tibia articular line. MJO and LJO were measured according to the same method at extension. The angle between the line in contact with the medial and lateral lowest points of the femoral prosthesis and the line in contact with the lower surface of the tibial prosthesis was measured; varus angle was defined as "varus ligament balance at flexion" (positive value in varus) (-Fig. 2B). The intrarater interclass correlation coefficient (ICC) for the MJOs and LJOs at extension was 0.931 (95\% confidence interval [CI]: 0.824-0.973) and 0.952 (95\% CI: 0.879-0.981), respectively. The intrarater ICC for the MJOs and LJOs at flexion was 0.947 (95\% CI: 0.866-0.979) and 0.944 (95\% CI: 0.857-0.978), respectively. These results indicate excellent reliability of the measurements.

\section{Statistical Analysis}

All values are presented as mean \pm standard error. Data analyses were performed using IBM SPSS statistics software (version 21; IBM Corp., Armonk, NY). The Shapiro-Wilk test was used to analyze normally distributed data using IBM SPSS statistics software. The unpaired $t$-test was performed to evaluate the differences in demographic data, femoral external rotation angle, and postoperative active range of motion (ROM) measured by lateral knee radiograph between the two techniques. Coronal FCA, TCA, and sagittal TCA were also evaluated using unpaired $t$-test between the two groups. The differences between MJO and LJO in each group were compared using a paired t-test. Each MJO and LJO was compared between the two techniques using one-way analysis of variance. Spearman's rank correlation analysis was conducted to assess the correlation between varus ligament balance at extension and flexion. A statistical priori power analysis was performed to determine the sample size based on the difference in $\mathrm{MJO}$ at 3 years postoperatively between the two techniques. In this analysis, G*Power software (version 3.1.9.2; Heinrich Heine Universität Düsseldorf, DE) was used with a prespecified significance level of $\alpha<0.05$, a power level of $95 \%$, and an effect size based on the results of the pilot study with 15 cases (effect size $d=0.63$ ). The estimated sample size was 55 patients. The post hoc power analysis for $\mathrm{MJO}$ at 3 years postoperatively further confirmed that the power was 0.95 . A $p$-value of $<0.05$ was set as the level of significance.

\section{Results}

\section{Femoral External Rotation Angle and Postoperative ROM}

A significant difference was found in the femoral external rotation angle between MPGT ( $4.2 \pm 0.2$ degrees) and MRT $(3.6 \pm 0.1$ degrees, $p<0.01)$. The mean active ROMs in the MPGT group were $-2.5 \pm 0.5-101.7 \pm 1.6$ degrees at 1 month, $-1.8 \pm 0.6-109.5 \pm 1.7$ degrees at 6 months, $-0.2 \pm 0.7-$ $111.2 \pm 1.5$ degrees at 1 year, and $0.5 \pm 0.6-112.0 \pm 1.7$ degrees at 3 years postoperatively. The mean active ROMs in the MRT group were $-1.9 \pm 0.7-104.4 \pm 1.8$ degrees at 1 month, $-0.7 \pm 1.0-111.7 \pm 1.9$ degrees at 6 months, $0.8 \pm 0.8-$ $111.5 \pm 1.8$ degrees at 1 year, and $0.6 \pm 0.9-112.9 \pm 1.9$ degrees at 3 years postoperatively. No significant difference was found in the postoperative active ROMs between the two techniques at any time point.

Postoperative Radiographic Assessment of Components Postoperative coronal FCA, TCA, and sagittal TCA in the MPGT group were $7.0 \pm 0.2$ degrees in valgus, $1.3 \pm 0.2$ degrees in varus, and $6.7 \pm 0.2$ degrees with posterior inclination. Postoperative coronal FCA, TCA, and sagittal TCA in the MRT group were $6.6 \pm 0.5$ degrees in valgus, $1.2 \pm 0.5$ degrees in varus, and $6.9 \pm 0.2$ degrees with posterior inclination. No significant difference was found in the postoperative coronal FCA, TCA, and sagittal TCA between the two techniques. 
Table 2 MJO and LJO at extension and flexion in each surgical technique

\begin{tabular}{|l|l|l|l|l|l|l|}
\hline & \multicolumn{2}{|l|}{ At extension } & & \multicolumn{2}{l|}{ At flexion } & \\
\hline MPGT group & MJO & LJO & $p$-Value & MJO & LJO & $p$-Value \\
\hline $1 \mathrm{mo}$ & $2.94 \pm 0.16$ & $3.58 \pm 0.22$ & $0.01^{\mathrm{a}}$ & $0.77 \pm 0.10$ & $1.85 \pm 0.22$ & $<0.01^{\mathrm{a}}$ \\
\hline $6 \mathrm{mo}$ & $2.63 \pm 0.14$ & $3.52 \pm 0.22$ & $<0.01^{\mathrm{a}}$ & $0.88 \pm 0.09$ & $2.14 \pm 0.22$ & $<0.01^{\mathrm{a}}$ \\
\hline $1 \mathrm{y}$ & $2.68 \pm 0.17$ & $3.72 \pm 0.20$ & $<0.01^{\mathrm{a}}$ & $0.98 \pm 0.09$ & $2.16 \pm 0.22$ & $<0.01^{\mathrm{a}}$ \\
\hline $3 \mathrm{y}$ & $2.76 \pm 0.18$ & $3.64 \pm 0.18$ & $<0.01^{\mathrm{a}}$ & $0.69 \pm 0.07$ & $2.04 \pm 0.27$ & $<0.01^{\mathrm{a}}$ \\
\hline MRT group & MJO & LJO & -Value & MJO & LJO & $p$-Value \\
\hline $1 \mathrm{mo}$ & $3.17 \pm 0.17$ & $4.21 \pm 0.23$ & $<0.01^{\mathrm{a}}$ & $1.09 \pm 0.13$ & $2.17 \pm 0.25$ & $<0.01^{\mathrm{a}}$ \\
\hline $6 \mathrm{mo}$ & $3.05 \pm 0.19$ & $4.02 \pm 0.23$ & $<0.01^{\mathrm{a}}$ & $1.38 \pm 0.16$ & $2.67 \pm 0.27$ & $<0.01^{\mathrm{a}}$ \\
\hline $1 \mathrm{y}$ & $3.23 \pm 0.21$ & $4.38 \pm 0.24$ & $<0.01^{\mathrm{a}}$ & $1.42 \pm 0.16$ & $2.72 \pm 0.30$ & $<0.01^{\mathrm{a}}$ \\
\hline $3 \mathrm{y}$ & $3.40 \pm 0.24$ & $4.45 \pm 0.26$ & $<0.01^{\mathrm{a}}$ & $1.15 \pm 0.16$ & $2.26 \pm 0.37$ & $<0.01^{\mathrm{a}}$ \\
\hline
\end{tabular}

Abbreviations: LJO, lateral joint opening; MJO, medial joint opening; MPGT, medial preserving gap technique; MRT, measured resection technique. ${ }^{\text {a }}$ Statistically significant MJO versus LJO.

\section{Medial and Lateral Joint Opening}

A smaller joint opening indicates a higher postoperative stability; the mean MJOs and LJOs at extension and flexion using each technique are listed in $\mathbf{-}$ Tables 2 and $\mathbf{3}$. MJOs both at extension and flexion using each technique were significantly smaller than the LJOs at all time points ( $\mathbf{- T a b l e ~} \mathbf{2}$ ). MJOs and LJOs at extension in the MPGT group were significantly smaller than those in the MRT group, suggesting that medial and lateral postoperative knee stability at extension was greater following MPGT, at 1 and 3 years postoperatively (-Table 3, -Fig. 3). Furthermore, MJOs at flexion in the MPGT group were significantly smaller than those in the MRT group, suggesting that medial postoperative knee stability at flexion was greater following MPGT, at 6 months, 1 year, and 3 years postoperatively ( $\mathbf{- T a b l e ~} \mathbf{3}$, $\mathbf{- \text { Fig. }} \mathbf{4}$ ). No significant difference in LJO at flexion was found between the two surgical techniques.

\section{Varus Ligament Balance}

The mean varus ligament balances at extension and flexion are listed in -Table 4. No significant correlation was found between varus ligament balance at extension and flexion in the MRT group at any time point; however, the varus ligament balance at extension was significantly correlated to the varus ligament balance at flexion in the MPGT group at all time points. This suggests that compared with the MRT group, the MPGT group was able to achieve the equal trapezoid gaps at extension and flexion.

\section{Discussion}

The most important finding of the present study was that compared with MRT, MPGT was better at preserving postoperative medial knee stability both at extension and flexion after PS-TKA, supporting our hypothesis. According to a recent development, MPGT focuses on medial stability and essentially involves making unequal rectangular gaps using the modified gap technique, but equal trapezoidal gaps at extension and flexion. ${ }^{6}$ Nagai et al reported that the varus ligament balance and joint center gaps, both at extension and flexion, increased with increased joint distraction force; however, the differences in varus ligament balance and joint

Table 3 Comparison of MJO and LJO at extension and flexion between two surgical techniques

\begin{tabular}{|l|l|l|l|l|l|l|}
\hline & \multicolumn{2}{|l|}{ At extension } & & \multicolumn{2}{l|}{ At flexion } & \\
\hline MJO & MPGT group & MRT group & $p$-Value & MPGT group & MRT group & $p$-Value \\
\hline $1 \mathrm{mo}$ & $2.94 \pm 0.16$ & $3.17 \pm 0.17$ & 0.31 & $0.77 \pm 0.10$ & $1.09 \pm 0.13$ & 0.06 \\
\hline $6 \mathrm{mo}$ & $2.63 \pm 0.14$ & $3.05 \pm 0.19$ & 0.08 & $0.88 \pm 0.09$ & $1.38 \pm 0.16$ & $<0.01^{\mathrm{a}}$ \\
\hline $1 \mathrm{y}$ & $2.68 \pm 0.17$ & $3.23 \pm 0.21$ & $0.03^{\mathrm{a}}$ & $0.98 \pm 0.09$ & $1.42 \pm 0.16$ & $0.02^{\mathrm{a}}$ \\
\hline $3 \mathrm{y}$ & $2.76 \pm 0.18$ & $3.40 \pm 0.24$ & $0.03^{\mathrm{a}}$ & $0.69 \pm 0.07$ & $1.15 \pm 0.16$ & $<0.01^{\mathrm{a}}$ \\
\hline LJO & MPGT group & MRT group & $p$-Value & MPGT group & MRT group & $p$-Value \\
\hline $1 \mathrm{mo}$ & $3.58 \pm 0.22$ & $4.21 \pm 0.23$ & 0.05 & $1.85 \pm 0.22$ & $2.17 \pm 0.25$ & 0.34 \\
\hline $6 \mathrm{mo}$ & $3.52 \pm 0.22$ & $4.02 \pm 0.23$ & 0.13 & $2.14 \pm 0.22$ & $2.67 \pm 0.27$ & 0.14 \\
\hline $1 \mathrm{y}$ & $3.72 \pm 0.20$ & $4.38 \pm 0.24$ & $0.04^{\mathrm{a}}$ & $2.16 \pm 0.22$ & $2.72 \pm 0.30$ & 0.13 \\
\hline $3 \mathrm{y}$ & $3.64 \pm 0.18$ & $4.45 \pm 0.26$ & $0.01^{\mathrm{a}}$ & $2.04 \pm 0.27$ & $2.26 \pm 0.37$ & 0.63 \\
\hline
\end{tabular}

Abbreviations: LJO, lateral joint opening; MJO, medial joint opening; MPGT, medial preserving gap technique; MRT, measured resection technique. ${ }^{\text {aS }}$ tatistically significant MPGT versus MRT. 


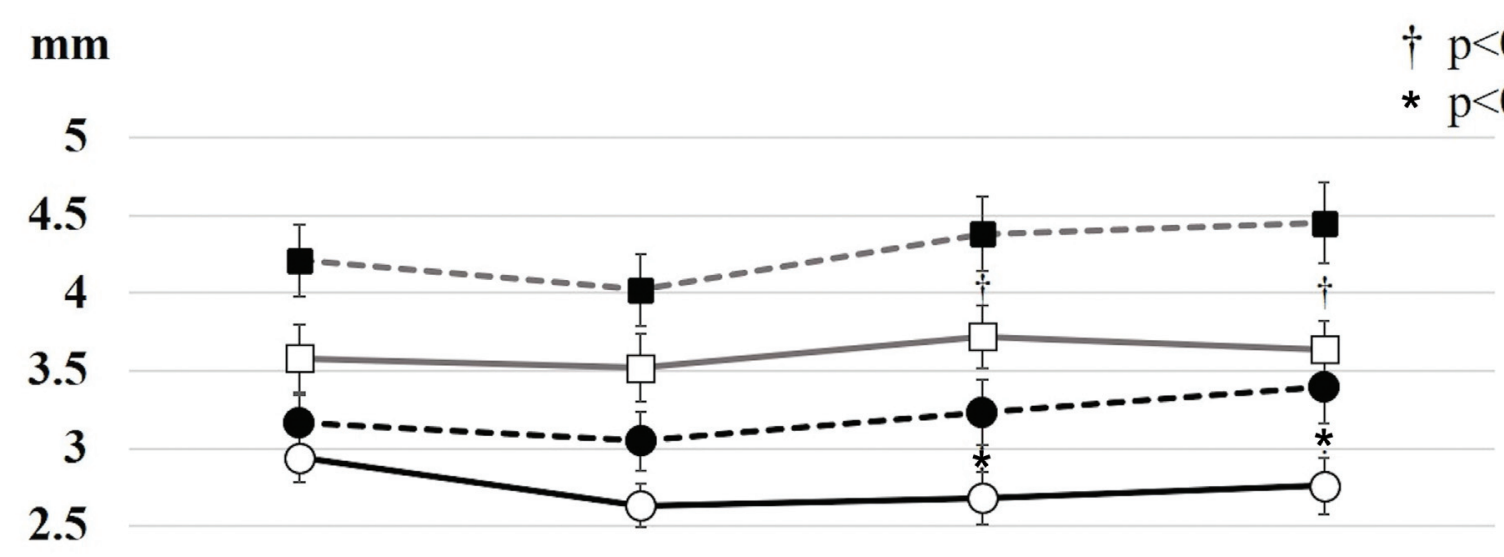

\section{2}

1.5

0.5

0

$\begin{array}{lrlr}1 \text { month } & 6 \text { months } & 1 \text { year } & 3 \text { years } \\ - \text {-MPGT group MJO } & - \text {-MRT group MJO } \\ -\square-\text { MPGT group LJO } & -\square-\text { MRT group LJO }\end{array}$

Fig. 3 Comparison of medial and lateral joint opening at extension between the two surgical techniques. Medial joint opening (MJO) and lateral joint opening (LJO) at extension in the medial preserving gap technique (MPGT) group were significantly smaller than those in the measured resection technique (MRT) group at 1 and 3 years postoperatively. $\dagger p<0.05$ MPGT group versus MRT group in LJO. ${ }^{*} p<0.05$ MPGT group versus MRT group in MJO.

center gap at extension and flexion were consistent among different joint distraction forces from 20 to $60 \mathrm{lbs}^{13}$ This novel index was considered useful to determine the femoral rotational angle and resection thickness of posterior femoral condyles in the modified gap technique; therefore, a novel surgical technique for varus type knee OA based on the modified gap technique and tensor measurement was devised, namely MPGT. The main concepts of MPGT are as follows: first, the procedure preserves medial knee stability both at extension and flexion but allows for lateral physiological laxity. This means that equal trapezoidal osteotomy gaps, both at extension and flexion, are aimed with varus ligament balance between the cutting surfaces of the femur and tibia. Second, this procedure provides a safe and quantitative surgical technique via the use of a tensor device and is therefore independent of the surgeon's experience. The femoral rotational angle and resection thickness of the posterior femoral condyles are based on the differences in the varus ligament balance and joint center gap between extension and flexion before posterior femoral condylar resections (-Fig. 1).

The rotational alignment of the femoral component can be determined in various ways; in the gap-balancing technique, the surgeon performs ligamentous releases to balance the knee at extension after the femoral and tibial cuts are per- formed. This is followed by resection of the posterior femoral condyle parallel to the prepared cut tibial surface by applying equal loads to the medial and lateral compartments. ${ }^{1,23}$ In MRT, a surface-derived reference axis of the femur is used as a guide to determine the position of the femoral component in the axial plane $^{2}$; several different reference axes derived from the bony landmarks have been introduced, of which the posterior condylar axis, ${ }^{14}$ Whiteside's line, ${ }^{16}$ and epicondylar axis ${ }^{17}$ are the most popular. Luyckx et al reported a tendency toward more femoral external rotation in the gap-balancing technique than in MRT. ${ }^{24}$ Lee et al also reported that the gap-balancing technique provided a more accurate and precise method for obtaining an adequate FCA than MRT during postoperative evaluations of the FCA. ${ }^{25}$ Additionally, the FCA obtained using MRT was significantly more internally rotated than that when using the gap-balancing technique. ${ }^{25}$ In this study, a small but significant difference was found in the femoral external rotation angle between the MPGT ( $4.2 \pm 0.2$ degrees $)$ and MRT (3.6 \pm 0.1 degrees) groups. This result supports the concept of MPGT, which is based on the modified gap technique; femoral rotational angle is based on the differences in the varus ligament balance between extension and flexion in MPGT.

Previous studies reported that medial and lateral ligamentous laxities were not balanced in normal knees; lateral ligamentous laxity was observed compared with medial 


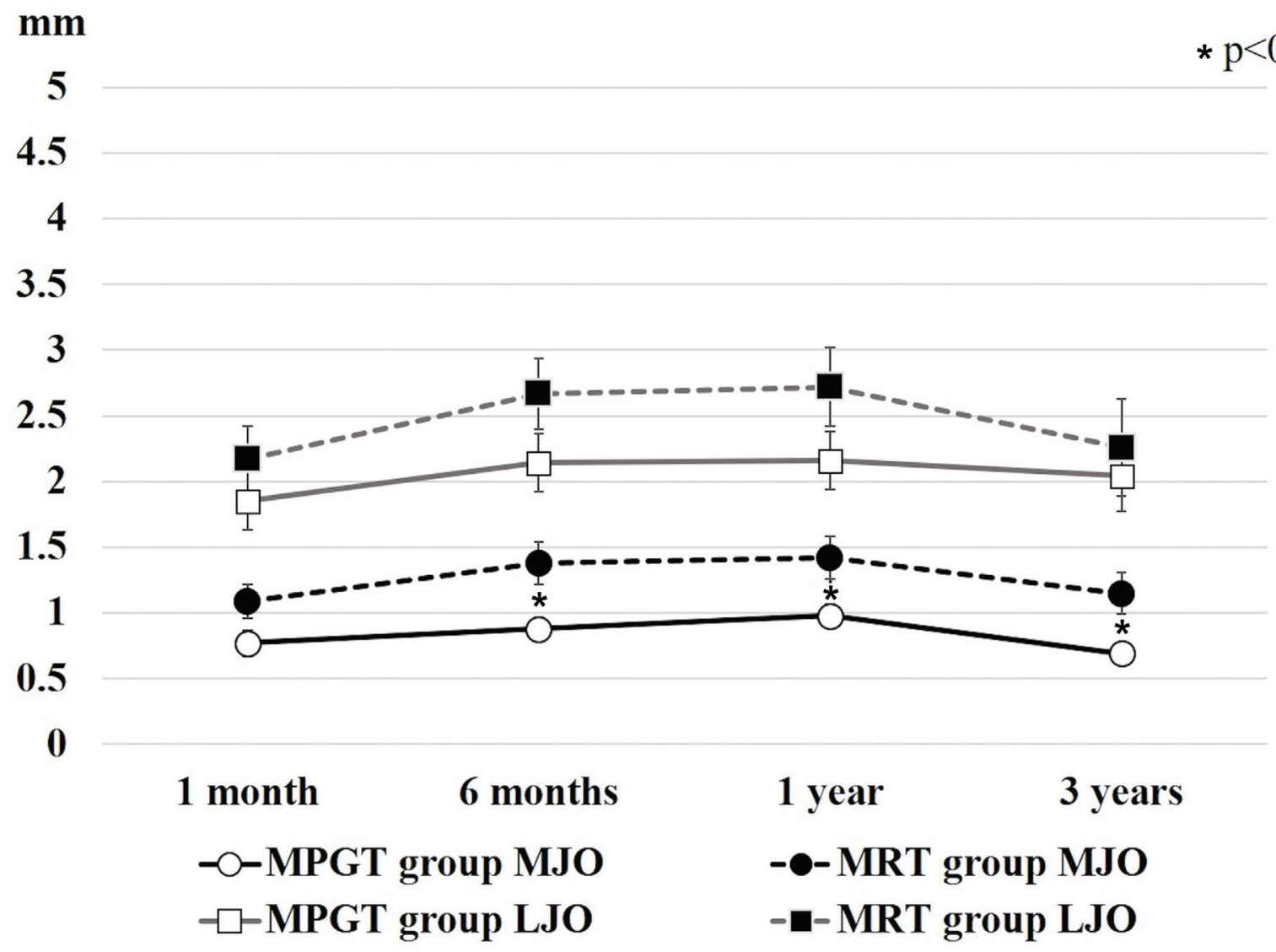

Fig. 4 Comparison of medial and lateral joint opening at flexion between the two surgical techniques. Medial joint opening (MJO) at flexion in the medial preserving gap technique (MPGT) group was significantly smaller than those in the measured resection technique (MRT) group at 6 months, 1 year, and 3 years postoperatively. No significant difference in lateral joint opening (LJO) at flexion was found between the two surgical techniques. ${ }^{*} p<0.05$ MPGT group versus MRT group in MJO.

ligamentous laxity. ${ }^{22,26}$ Medial stability with relative lateral laxity was also reported to be associated with good functional outcomes after well-aligned TKA. ${ }^{27}$ Furthermore, several reports indicated that medial instability after TKA may cause postoperative knee pain, ${ }^{4}$ more abnormal kinematics including anteroposterior instability, ${ }^{5,28}$ and poor functional outcomes. ${ }^{27}$ MPGT aims to preserve the medial stability of the knee both at extension and flexion while allowing for lateral physiological laxity. In this study, MJOs in the MPGT group, both at extension and flexion, were significantly smaller than the LJOs at all time points, supporting the aim of MPGT.

MJOs in the MPGT group, both at extension and flexion, were significantly smaller than those in the MRT group at 1 and 3 years postoperatively, suggesting that medial postoperative knee stability both at extension and flexion was greater than that when using MPGT. The medial soft tissue release was different between MPGT and MRT in this study. Using MPGT, the medial soft tissue release was conservatively performed via femoral and tibial osteophyte removal and deep MCL release, within $1 \mathrm{~cm}$ from the medial tibial plateau until a $10-\mathrm{mm}$ spacer block could be inserted, allowing physiological lateral laxity. On the other hand, using MRT, medial soft tissue balance was performed via femoral and tibial osteophyte removal, as well as deep layer MCL, medial, and posteromedial capsule release along the medial knee structures. Furthermore, additional soft tissue release (e.g., superficial layer of $\mathrm{MCL}$ ) was performed to adjust the soft tissue balance in the coronal plane while evaluating balance using a 10-mm spacer block. Yagishita et al reported that release of the medial and posteromedial capsule increases both medial and lateral joint gaps in extension, and particularly, in flexion. ${ }^{29}$ Mullaji et al also reported that although deep MCL release has a negligible effect, superficial MCL release increases the medial joint gap in extension, and particularly, in flexion. ${ }^{30}$ In this study, although there was a chronological change of soft tissue tension, medial soft tissue release during MPGT was more limited than that during MRT, as described above; as a result, MJOs in the MPGT group at extension-and especially at flexion-were significantly smaller than those in the MRT group at 1 and 3 years postoperatively, which also supports the concept of MPGT.

The modified gap technique is advocated to obtain equal mediolateral soft-tissue balance and rectangular parallel gaps both in extension and flexion. ${ }^{31}$ However, the risk of medial instability arises in many cases when aiming for the perfect parallel ligament balance of the knee at extension. MPGT focuses on medial stability, aiming for equal trapezoidal gaps in extension and flexion and allowing for lateral physiological laxity. In this study, no significant correlation 


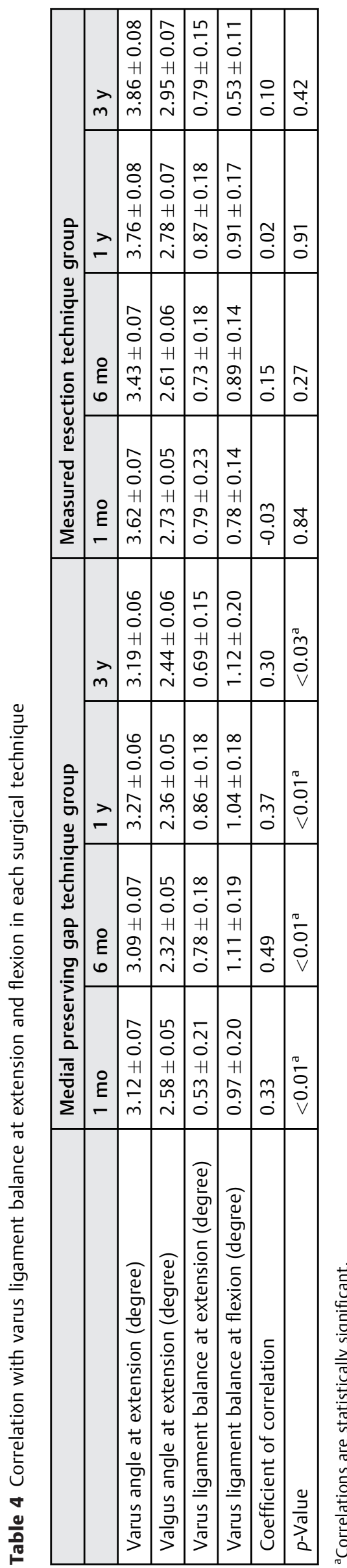

was found between varus ligament balance at extension and that at flexion in the MRT group at any time points. However, the varus ligament balance at extension was significantly correlated to the varus ligament balance at flexion in the MPGT group at all time points. This was because equal trapezoidal gaps in extension and flexion were accomplished in MPGT according to the preserving medial stability while allowing for lateral physiological laxity.

The present study has some limitations. First, the study population was almost composed of women and limited to patients with varus type $O A$ and excluded those with severe osteoarthritic knees with large bony defects. Conducting research in a group with equal proportions of each sex and valgus and severe osteoarthritic knees and comparing with the populations in this series are necessary for future investigations. Second, an implant with dual radius curvature was used in this study, which might have influenced the results. Different prosthetic types should be examined in the future. Third, this was a retrospective study. Furthermore, no clinical instruments were reported and the relationships between postoperative knee stabilities and functional outcomes were not evaluated. In future studies, patient-reported subjective outcomes, functional outcomes, radiographic parameters, and complication rates should be investigated. Forth, femoral component alignment in the sagittal and axial planes and tibial component alignment in the axial plane were not investigated in this study. Finally, the two techniques were not randomized and were performed during different periods. However, each surgery was performed by an experienced single surgeon, and the number of subjects was larger based on the power analysis; hence, the bias would be minimal.

\section{Conclusions}

This study investigated the differences in postoperative knee stability after PS-TKA with MPGT and MRT. MPGT achieved better postoperative medial knee stabilities both at extension and flexion than MRT even at 3 years after PS-TKA. MPGT is a more feasible method for preserving postoperative medial knee stability than MRT after PS-TKA, which would help avoid postoperative knee pain, abnormal kinematics including anteroposterior instability, and poor functional outcome; caused by postoperative medial instability after TKA.

Conflict of Interest

None declared.

\section{References}

1 Hungerford DS. Measured resection: a valuable tool in TKA. Orthopedics 2008;31(09):941-942

2 Dennis DA. Measured resection: an outdated technique in total knee arthroplasty. Orthopedics 2008;31(09):940,943-944

3 Matsumoto T, Muratsu H, Kawakami Y, et al. Soft-tissue balancing in total knee arthroplasty: cruciate-retaining versus posteriorstabilised, and measured-resection versus gap technique. Int Orthop 2014;38(03):531-537

4 Liebs TR, Kloos S-A, Herzberg W, Rüther W, Hassenpflug J. The significance of an asymmetric extension gap on routine 
radiographs after total knee replacement: a new sign and its clinical significance. Bone Joint J 2013;95-B(04):472-477

5 Nakamura S, Ito H, Yoshitomi H, Kuriyama S, Komistek RD, Matsuda S. Analysis of the flexion gap on in vivo knee kinematics using fluoroscopy. J Arthroplasty 2015;30(07):1237-1242

6 Tsubosaka M, Muratsu H, Takayama K, Miya H, Kuroda R, MatsumotoT. Comparison of intraoperative soft tissue balance between cruciate-retaining and posterior-stabilized total knee arthroplasty performed by a newly developed medial preserving gap technique. J Arthroplasty 2018;33(03):729-734

7 Nagai K, Muratsu H, Kanda Y, et al. Intraoperative soft tissue balance using novel medial preserving gap technique in posteriorstabilized total knee arthroplasty: comparison to measured resection technique. Knee Surg Sports Traumatol Arthrosc 2018;26 (11):3474-3481

8 Kamenaga T, Muratsu H, Kanda Y, Miya H, Kuroda R, Matsumoto T. The influence of postoperative knee stability on patient satisfaction in cruciate-retaining total knee arthroplasty. J Arthroplasty 2018;33(08):2475-2479

9 Iranpour-Boroujeni T, Li J, Lynch JA, Nevitt M, Duryea JOAI Investigators. A new method to measure anatomic knee alignment for large studies of OA: data from the osteoarthritis initiative. Osteoarthritis Cartilage 2014;22(10):1668-1674

10 MatsumotoT, Muratsu H, Tsumura N, et al. Joint gap kinematics in posterior-stabilized total knee arthroplasty measured by a new tensor with the navigation system. J Biomech Eng 2006;128(06): 867-871

11 Matsumoto T, Kuroda R, Kubo S, Muratsu H, Mizuno K, Kurosaka $M$. The intra-operative joint gap in cruciate-retaining compared with posterior-stabilised total knee replacement. J Bone Joint Surg Br 2009;91(04):475-480

12 Muratsu H, Matsumoto T, Kubo S, et al. Femoral component placement changes soft tissue balance in posterior-stabilized total knee arthroplasty. Clin Biomech (Bristol, Avon) 2010;25 (09):926-930

13 Nagai K, Muratsu H, Matsumoto T, Miya H, Kuroda R, Kurosaka M. Soft tissue balance changes depending on joint distraction force in total knee arthroplasty. J Arthroplasty 2014;29(03):520-524

14 Hungerford DS, Kenna RV. Preliminary experience with a total knee prosthesis with porous coating used without cement. Clin Orthop Relat Res 1983;(176):95-107

15 Arima J, Whiteside LA, McCarthy DS, White SE. Femoral rotational alignment, based on the anteroposterior axis, in total knee arthroplasty in a valgus knee. A technical note. J Bone Joint Surg Am 1995;77(09):1331-1334

16 Whiteside LA, Arima J. The anteroposterior axis for femoral rotational alignment in valgus total knee arthroplasty. Clin Orthop Relat Res 1995;(321):168-172

17 Berger RA, Rubash HE, Seel MJ, Thompson WH, Crossett LS. Determining the rotational alignment of the femoral component in total knee arthroplasty using the epicondylar axis. Clin Orthop Relat Res 1993;(286):40-47

18 Yoshino N, Takai S, Ohtsuki Y, Hirasawa Y. Computed tomography measurement of the surgical and clinical transepicondylar axis of the distal femur in osteoarthritic knees. J Arthroplasty 2001;16 (04):493-497

19 Ishii Y, Matsuda Y, Ishii R, Sakata S, Omori G. Coronal laxity in extension in vivo after total knee arthroplasty. J Orthop Sci 2003;8 (04):538-542

20 Matsumoto T, Muratsu H, Kubo S, Matsushita T, Kurosaka M, Kuroda R. Intraoperative soft tissue balance reflects minimum 5-year midterm outcomes in cruciate-retaining and posterior-stabilized total knee arthroplasty. J Arthroplasty 2012;27(09):1723-1730

21 Kanekasu K, Kondo M, Kadoya Y. Axial radiography of the distal femur to assess rotational alignment in total knee arthroplasty. Clin Orthop Relat Res 2005;(434):193-197

22 Tokuhara Y, Kadoya Y, Kanekasu K, Kondo M, Kobayashi A, Takaoka K. Evaluation of the flexion gap by axial radiography of the distal femur. J Bone Joint Surg Br 2006;88(10):1327-1330

23 Scuderi GR, Insall JN. The posterior stabilized knee prosthesis. Orthop Clin North Am 1989;20(01):71-78

24 Luyckx T, Peeters T, Vandenneucker H, Victor J, Bellemans J. Is adapted measured resection superior to gap-balancing in determining femoral component rotation in total knee replacement? J Bone Joint Surg Br 2012;94(09):1271-1276

25 Lee JK, Lee S, Chun SH, Kim KT, Lee MC. Rotational alignment of femoral component with different methods in total knee arthroplasty: a randomized, controlled trial. BMC Musculoskelet Disord 2017;18(01):217

26 Okazaki K, Miura H, Matsuda S, et al. Asymmetry of mediolateral laxity of the normal knee. J Orthop Sci 2006;11(03):264-266

27 Aunan E, Kibsgård TJ, Diep LM, Röhrl SM. Intraoperative ligament laxity influences functional outcome 1 year after total knee arthroplasty. Knee Surg Sports Traumatol Arthrosc 2015;23 (06):1684-1692

28 Nakamura S, Kuriyama S, Nishitani K, Ito H, Murata K, Matsuda S. Correlation between intraoperative anterior stability and flexion gap in total knee arthroplasty. J Arthroplasty 2018;33(08): 2480-2484

29 Yagishita K, Muneta T, Ikeda H. Step-by-step measurements of soft tissue balancing during total knee arthroplasty for patients with varus knees. J Arthroplasty 2003;18(03):313-320

30 Mullaji A, Sharma A, Marawar S, Kanna R. Quantification of effect of sequential posteromedial release on flexion and extension gaps: a computer-assisted study in cadaveric knees. J Arthroplasty 2009;24(05):795-805

31 Moro-oka TA, Shiraishi H, Iwamoto Y, Banks SA. Modified gapbalancing technique in total knee arthroplasty: evaluation of the post-operative coronal laxity. Knee Surg Sports Traumatol Arthrosc 2010;18(03):375-380 